\title{
Integrating fiber optics into electronic communications curriculum
}

\section{Shuping Wang}

Shuping Wang, "Integrating fiber optics into electronic communications curriculum," Proc. SPIE 11143, Fifteenth Conference on Education and Training in Optics and Photonics: ETOP 2019, 111430P (2 July 2019); doi: $10.1117 / 12.2520771$

SPIE Event: Fifteenth Conference on Education and Training in Optics and Photonics: ETOP 2019, 2019, Quebec City, Quebec, Canada 


\title{
Integrating fiber optics into electronic communications curriculum
}

\author{
Shuping Wang \\ College of Engineering, University of North Texas, 3940 N. Elm St., TX, USA 76207
}

\begin{abstract}
Traditional electronic communications curriculum spends large amount of time to cover the electronic circuits for communications. Since highly integrated circuits for communications are now available, in many cases a blockdiagram level analysis is sufficient. On the other hand, fiber optics for communications has been used not only for long haul transmission of information, but also local area networks and computer networks. To stay relevant and keep up with industry trends without adding new courses, we revised existing two-semester electronic communications curriculum by replacing some circuit details with topics on optical fiber communications fundamentals and wavelength division multiplexing techniques. Since optical fiber communications itself can be a course of one or two semesters, we are not intending to cover the topic in all aspects. Instead, our goal is to introduce the highlights of the major topics necessary for students to understand basics of optical fiber communications. To make the topic transition logically we introduced the light source in fiber optics topic, after establishing the modulation/demodulation concept, as an alternative carrier signal for modulation process. We then introduced optical fibers as transmission channel for modulated optical carriers, followed by other components (optical sources, detectors, and etc.). Wavelength division multiplexing had also been introduced as one of the major multiplexing techniques, after introducing the time division multiplexing and frequency division multiplexing concepts, for both long haul and high speed Ethernet applications. Through the midterm/final examinations students demonstrated similar level of understanding of the traditional materials as before. At the same time their basic knowledge on optical fiber communications were established.
\end{abstract}

Keywords: Fiber optics, optical fiber communications, electronic communications curriculum

\section{INTRODUCTION}

The electronic communications curriculum, in the Department of Engineering Technology at the University of North Texas, includes two courses: Electronic Communications I and II. The prerequisites for the Electronic Communications courses are the Circuit Analysis and the Advanced Circuit Analysis. Fundamental circuit analysis methods and the important topics for electronic communications, such as filters, tuned circuits, and Fourier transform, are covered thoroughly in the circuit analysis courses.

Since highly integrated circuits (ICs) for electronic communications are now available, in many cases a blockdiagram level analysis of the circuits is sufficient for their applications in the electronic communications courses. The monolithic function generator IC XR-2206 manufactured by EXAR Corporation is one of the examples. The output of the XR-2206 can be both amplitude and frequency modulated by an external signal. In this case, the detailed circuit analysis of different kinds of oscillators/carriers could be replaced by a block diagram, and the emphasis is placed on the establishment of modulation/demodulation concepts. On the other hand, fiber optics for communications has been used not only for long haul transmission of information, but also local area networks and computer networks. To stay relevant and keep up with industry trends without adding new courses, we revised existing two-semester electronic communications curriculum by replacing some circuit analysis details with topics on optical fiber communications fundamentals and wavelength division multiplexing techniques. Since optical fiber communications itself can be a course of one or two semesters, we are not intending to cover the topic in all aspects. Instead, our objective is to introduce the highlights of the major topics necessary for students to understand basics of optical fiber communications. 
To make the topic transition logically we introduced light sources in fiber optics topic, after establishing the modulation/demodulation concept, as an alternative carrier signal for modulation process. We then introduced optical fiber as the transmission channel for modulated optical carriers, followed by other components (optical sources, detectors, and etc.). Wavelength division multiplexing has also been introduced as one of the major multiplexing techniques, after introducing the time division multiplexing and frequency division multiplexing concepts, for both long haul and high speed Ethernet applications. Rest of the paper will describe in detail how the curriculum was revised.

\section{CURRICULUM REVISION}

Because of the availability of highly integrated circuits for communications as mentioned in the Introduction, we have eliminated the entire chapter on electronic fundamentals for communications from the Electronic Communications I course ELET4710. The elimination allows us to allocate two to three weeks for addition of fiber optics contents. This revision doesn't affect the quality and the flow of the course due to the following two reasons: 1) As pointed out in the introduction, prerequisite for ELET4710 is ELET3700 Advanced Circuit Analysis that covers most of the basic communication electronic circuits operation (including oscillators, amplifiers, filters, tuned circuits, analog-to-digital and digital-to-analog converters, etc.);2) We dedicated lab sessions in the first three weeks to filters and tuned circuits, serving the purpose of reviewing and academic leveling of the electronic fundamentals for communications. With the saved time, we were able to build fiber optical communication contents into the curriculum through the approaches described in sections 2.1 and 2.2 respectively.

\subsection{Blending fiber optical communications concept throughout the semesters}

In the introduction chapter, a general model of all communication systems, as shown in Figure 1, with three basic components (i.e. the transmitter, the transmission medium, and the receiver) was introduced. The function of a transmitter is to convert the information to be transmitted into a signal that is compatible with the transmission medium. The conversion is involved in a process of modulation in which the information to be transmitted modulates a carrier signal. Depending on the type of the transmission medium, a suitable carrier is generated inside the transmitter. The role of the receiver is to remove the information to be transmitted from the carrier and recover the information through the demodulation process. At this point, optical fiber as one type of the transmission mediums is introduced first time, meaning a carrier signal that is compatible with optical fiber is necessary. Naturally an optical source (laser/light emitting diode (LED)) as the information carrier, and an optical detector (photodiode) as part of the demodulator inside the receiver are necessary and therefore were briefly deliberated at this point. Detailed discussions were covered in the chapter that was added by the curriculum revision and dedicated to optical communications.

\section{Communication Systems}

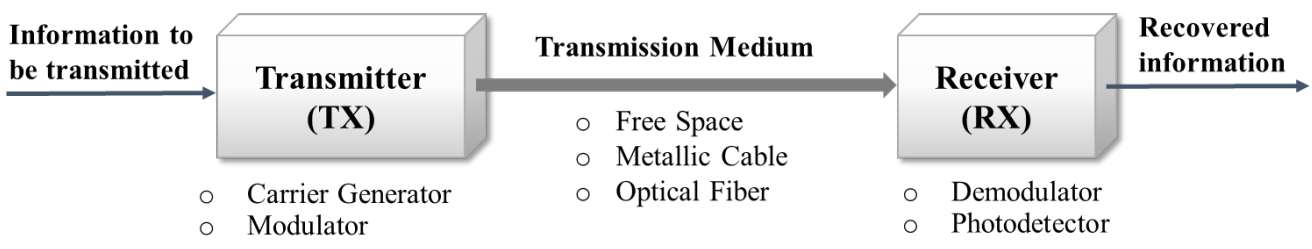

Figure 1. A generic model of all communication systems

In the chapter that covers the analog and digital modulation/demodulation, an optical signal as a carrier in the modulation/demodulation process was introduced. After the presentation of the amplitude shift keying (ASK) where a binary data modulates a radio frequency carrier, as an analogy of ASK, the optical modulation of the on-off keying (OOK), i.e. using binary data to be transmitted to modulate laser/LED beam was presented. 
In order to increase the transmission capacity with existing transmission medium channels, multiplexing technique, as shown in Figure 2, has been implemented where two or more signals are transmitted simultaneously. A multiplexer combines multiple input signals into one composite signal and is then transmitted over a single transmission medium. A demultiplexer is used to separate individual component signals at the output. One of the most common types of multiplexing is frequency division multiplexing (FDM) in which individual signals are assigned to different frequencies (subcarriers) within a common bandwidth. Modulated subcarriers are then multiplexed and transmitted through a single transmission medium. Since the wavelength division multiplexing (WDM) is actually same as the FDM, but operating over the light wave bandwidth, we extended the multiplexing chapter by including WDM for the situation where the transmission medium is optical fiber. The subcarriers in FDM were replaced by the component wavelengths (optical subcarriers) in WDM.

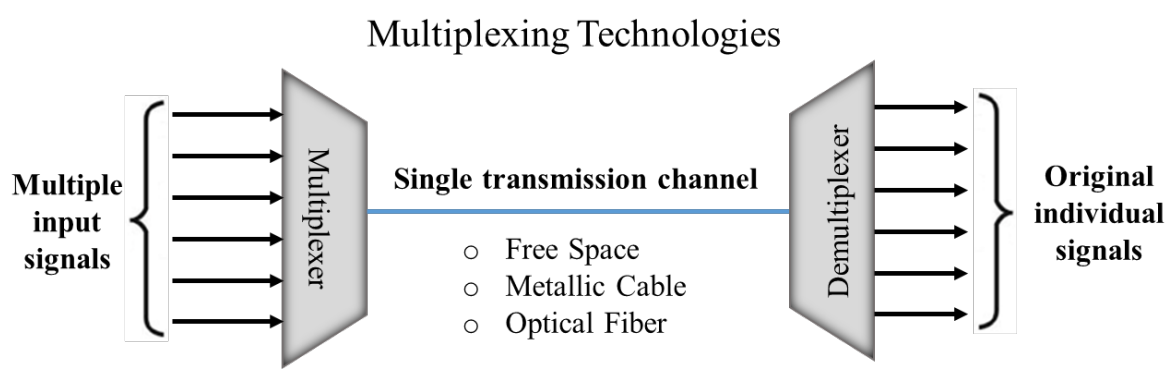

Figure 2. Concept of multiplexing/demultiplexing

Dense wavelength division multiplexing (DWDM) was introduced as an application example to long-haul sectors to meet the increased bandwidth demands. Instead of replacing existing fiber that is normally costly, DWDM technology is a better option to add channel capacity and thus upgrade the lifespan of a network. DWDM based on bulk-optic diffraction gratings as depicted in Figure 3 was used to explain how the multiplexing/demultiplexing is realized. Incident light of multiple wavelengths strikes the diffraction grating surface, the diffraction grating spatially separates light of different wavelengths as shown in the figure. The multiplexer or demultiplexer is obtained by placing the input and output optics (e.g. optical fibers) at the right positions for the desired wavelengths. The WDM based on diffraction grating is particularly suitable for high channel count systems because the insertion loss doesn't depend on the number of channels.

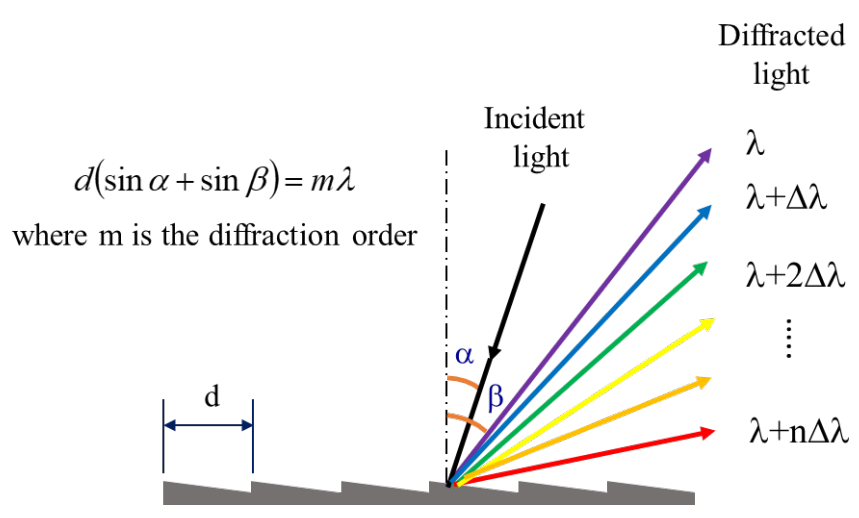

Figure 3 . The incident light is diffracted into all primary wavelengths by a bulk-optic diffraction grating

As an application example of the WDM to high speed Ethernet, coarse wavelength division multiplexing (CWDM) has been embedded within existing curriculum with minimal revision. CWDM and DWDM share the same multiplexing mechanism but differ in number of channels, channel spacing, and the ability to amplify the 
multiplexed signals in the optical space. CWDM has fewer channels and larger channel spacing compared to DWDM that has been used mainly in long hall and ultra-long hall backbone networks. Because wideband optical amplification for CWDM is not available, only up to tens of kilometers' optical propagations can be achieved. Therefore, CWDM has a good fit to access networks and many metro/regional networks. Figure 4 depicts a 4channel CWDM as one of the options to realize the advanced Ethernet. Four externally modulated distributed feedback (DFB) laser beams enter a CWDM multiplexer through four bandpass filters. Each filter allows one wavelength pass and reflects others as shown in the figure. By choosing a proper angle of the glass block with a mirror coated on its $2^{\text {nd }}$ surface, the transmitted and reflected beams are combined and focused into a single mode fiber (SMF) through a fiber coupling lens.

\section{CWDM to Achieve Advanced Ethernet}

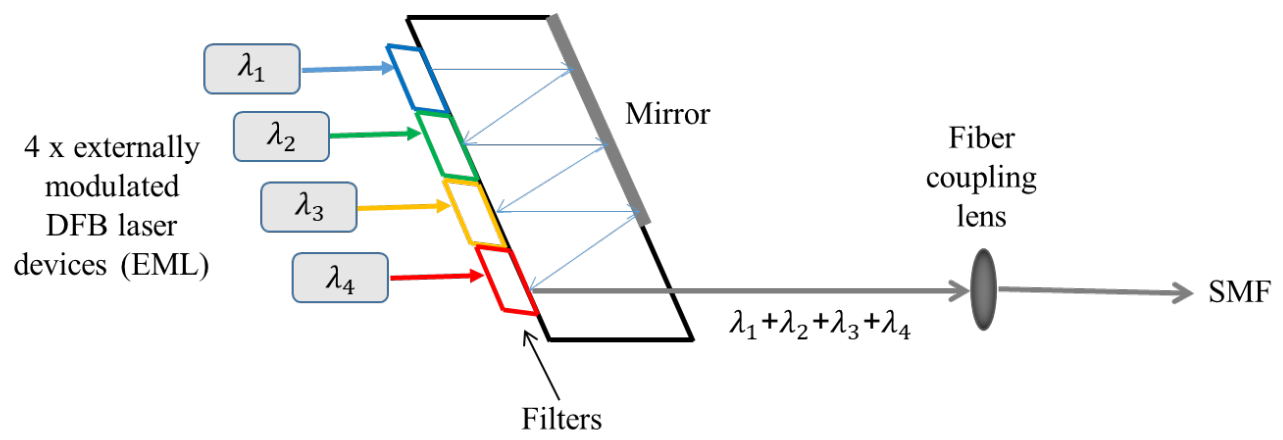

Figure 4. Schematic of a 4-channel CWDM multiplexer that combines four wavelengths and couples the multiplexed light signal into a single mode fiber (SMF).

\subsection{Introducing fiber optical communications system through a dedicated chapter}

The major topics for the revised two-semester curriculum is listed in Table 1. The optics-related contents described in section 2.1 were blended into the appropriate chapters where they are naturally belonged to. Further, we dedicated one chapter to optical fiber communications that was taught at the beginning of the second semester. We introduced the highlights of the major topics necessary for students to understand the basics of optical fiber communications as described in the rest of this section.

Table 1. Major topics covered by the revised curriculum

\begin{tabular}{|l||l|}
\hline \multicolumn{1}{|c||}{ Electronic Communications I } & \multicolumn{1}{|c|}{ Electronic Communications II } \\
\hline Introduction to Electronic/optical Communications & Optical Communications \\
\hline Amplitude Modulation/demodulation Fundamentals & Multiplexing and Demultiplexing - TDM, FDM, and WDM \\
\hline Fundamentals of Frequency Modulation/Demodulation & Fundamentals of Networking, Local-Area Networks, and Ethernet \\
\hline Communication Transmitters and Receivers & Digital Data Transmission \\
\hline
\end{tabular}

- Basic optical laws and definitions

Index of refraction - a fundamental optical parameter of a material, the concepts of reflection and refraction, and the Snell's law that determines the angles of reflected and refracted rays when an incident ray strikes the interface separating two different materials were reviewed first. An overview of the definition of critical angle and the concept of total internal reflection were given. 
- Optical fiber structure

Optical fiber structure characteristics determine the transmission properties of the fiber i.e. the way a light propagates along the optical fiber is affected by the fiber structure. A brief overview of optical fiber types categorized by optical fiber modes and index profiles were conducted. Most commonly used optical fiber types (i.e. single mode and multimode step-index and multimode graded-index fibers) were introduced, and their pros and cons were discussed.

\section{- Ray optics representation}

The geometrical optics approach was used to explain the propagation characteristics of light in an optical fiber. A step-index optical fiber as shown in Figure 5 was used to introduce the concept of acceptance angle $\theta_{A}$ for an optical fiber and its numerical aperture $N A$ in terms of the refractive indices of the optical fiber core and cladding. By applying Snell's law at the two interfaces (air-to-core and core-to-cladding) and the concept of total internal reflection, the NA for an optical fiber as shown in Figure 5 can be obtained:

$$
N A=\sin \theta_{A}=\sqrt{n_{1}^{2}-n_{2}^{2}}
$$

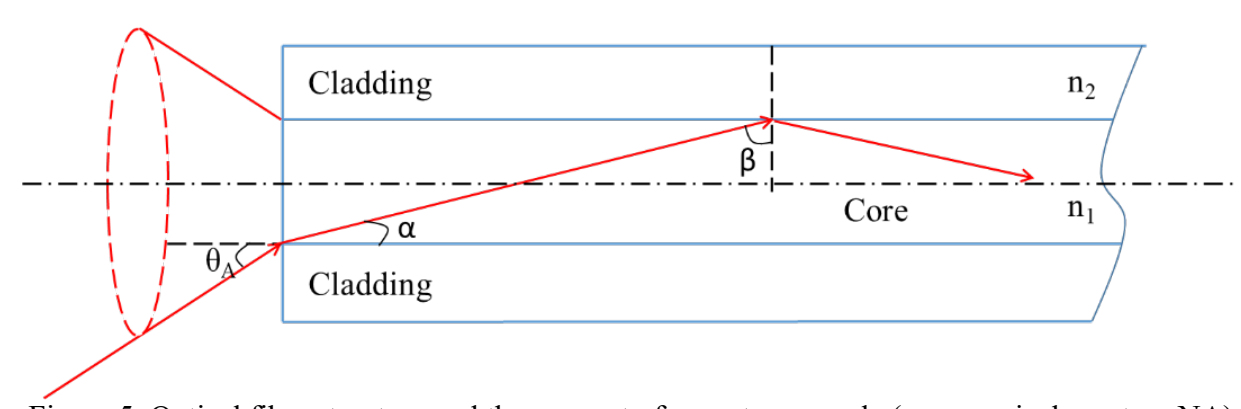

Figure 5. Optical fiber structure and the concept of acceptance angle (or numerical aperture NA)

- Optical transmitter/receiver operation (including optical sources and photodetectors)

An optical transmitter consists of a carrier generator and a modulator. A simple optical transmitter is a light source (an LED or a laser diode). The light beam is turned on/off (i.e. be modulated) by a binary pulse that is the signal that needs to be transmitted (i.e. modulating signal). As an example, a simple optical transmitter using a laser/LED in an optical communication system as shown in Figure 6 was used to explain the concepts. The critical component of an optical receiver is the light sensitive detector that converts the light pulses back to electrical pulses. A photodiode as shown in the figure was introduced as the photodetector in the optical receiver. The working mechanisms for LED and laser diode, their constructions, and radiation patterns were introduced in this section as well.

The OOK as shown in Figure 6 is only suitable for low data rate application, but it's an easy-to-understand way to present the optical modulation/demodulation concepts and therefore was used for that purpose. For higher data rate transmission, the indirect/external modulation methods were presented as well. As an example, the Mach-Zehnder (MZ) interferometer - one of the most popular electro-optical modulators (EOMs) was brought into the classroom. After the explanation of the working principle, the particular emphasize was given to the understanding of how the signal that needs to be transmitted (i.e. the modulating signal) is carried by the light beam (i.e. the carrier) in the amplitude modulation format. 


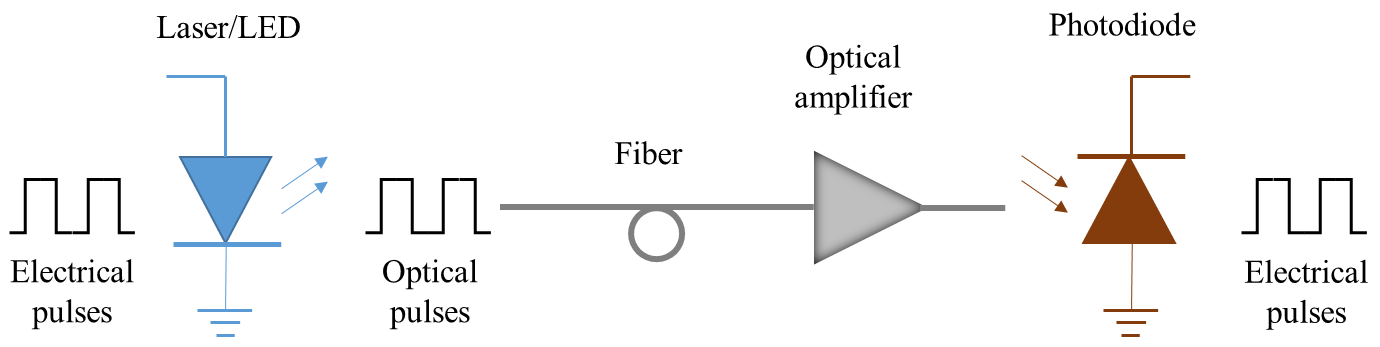

Figure 6. Schematic of optical communication system. The laser serves as the optical transmitter and the photodiode serves as the optical receiver.

\section{SUMMARY}

The fully revised version of the two-semester courses has been taught once and student responses are positive. Through the midterm/final examinations, students demonstrated similar level of understanding of the traditional materials as before. At the same time their basic knowledge on optical fiber communications are established, achieving the objective of the curriculum revision. Making the connection between the optical communications and the electronic communications helped students to grasp the optical modulation/demodulation easily and to appreciate the role the light plays in communication fields. 